\title{
Antilambda enhancement in ultrarelativistic heavy ion collisions
}

\author{
C. M. Ko, M. Asakawa, and P. Lévai* \\ Cyclotron Institute and Physics Department, Texas A $6 M$ University, College Station, Texas 77843
}

(Received 6 May 1992)

\begin{abstract}
In the Walecka model, the antilambda mass in dense nuclear matter is smaller than its value in free space. This reduces the threshold for antilambda production in dense matter that forms in the compression stage of ultrarelativistic heavy ion collisions. Because of the large number of mesons produced in the collision, the process $K M \rightarrow \bar{\Lambda} N$, where $M$ denotes either a pion or a rho meson, is shown to be important and provides thus a plausible explanation for the observed enhancement of antilambda yield in recent experiments carried out at CERN SPS with nuclear beams.
\end{abstract}

PACS number(s): 25.75.+r

In heavy ion collisions at ultrarelativistic energies, the quark-gluon plasma may be formed in the initial high energy density stage [1]. A number of signatures have been suggested for the detection of this state of matter. Among them is the enhanced production of strangeness as a result of the large abundance of strange quarks and antiquarks in the quark-gluon plasma [2]. This is based on the belief that strange particle production is suppressed in normal hadronic processes. However, it has been shown recently that this need not be the case because secondary interactions of the created particles can also produce strange particles and lead to strangeness enhancement $[3,4]$. This will particularly be so if one takes into account the change of hadron masses in the medium. Indeed, if one lets kaon mass decrease in dense matter as a result of the precursory effect of kaon condensation at high densities $[5,6]$, then kaon production from the process $\pi \pi \rightarrow K \bar{K}$ is enhanced and it can account for the experimentally measured enhancement of $K^{+} / \pi^{+}$ ratio in Brookhaven AGS experiments [7]. Similarly, the reduced phi meson mass in dense matter $[8,9]$ can also explain the large $\phi / \omega$ ratio observed in the CERN SPS experiments [10].

In the NA35 experiment of $\mathrm{S}+\mathrm{S}$ at $200 \mathrm{GeV} /$ nucleon [11], the antilambda yield is 1.5 per event and is 115 times greater than that in $p-p$ collisions at the same energy. Compared with the 36 -fold enhancement of the negatively charged particles, most of them being negative pions, there is a factor three enhancement of antilambda yield in heavy ion collisions. This enhancement can probably be explained if one assumes that a quark-gluon plasma is formed in the initial stage of the collisions. However, the formation of the quark-gluon plasma is unlikely for collisions between light nuclei such as the sulfur. Other explanations have also been proposed. Aichelin and Werner have emphasized the importance of the many-body effect [12]. Sorge et al. [13] have shown that the color rope formation in string excitations

\footnotetext{
${ }^{*}$ On leave from KFKI, Budapest, Hungary.
}

leads also to enhanced production of antilambdas. In this paper, we shall show that this enhancement can be accounted for by the lower antilambda production threshold as a result of the reduced antilambda mass in dense matter.

The decrease of lambda mass in dense matter is expected from the partial restoration of chiral symmetry in hot dense matter but has not been studied in detail. In Ref. [14], the Walecka model has been generalized to include the lambda particle. The in-medium lambda mass in this model is given by

$$
m_{\Lambda}^{*}=m_{\Lambda}-\frac{g_{\sigma \Lambda \Lambda} g_{\sigma N N}}{m_{\sigma}^{2}} \rho_{s},
$$

where $\rho_{s}$ is the nuclear scalar density and increases with nuclear density. The scalar meson mass is taken to be $m_{\sigma} \approx 550 \mathrm{MeV}$. According to the quark model [14], the lambda-scalar meson coupling constant $g_{\sigma \Lambda \Lambda}$ is about two-thirds of the nucleon-scalar meson coupling constant $g_{\sigma N N}$. We take $g_{\sigma N N}^{2} \approx 48$, which is about a factor two smaller than the value used in the normal Walecka model, to obtain an in-medium nucleon mass of about 0.8 times its mass in free space, which is close to the empirically determined value [15]. In Fig. 1, the density dependence of both the nucleon and lambda masses is shown. It shows that both masses decrease substantially in dense nuclear matter. The density dependence is especially strong below $3 \rho_{0}$, where $\rho_{0}$ is the normal nuclear matter density. Since the antilambda has the same mass as the lambda, its mass is reduced in dense matter as well.

In hadronic matter, the antilambda can be produced from the nucleon-nucleon interaction $(N N \rightarrow N N \Lambda \bar{\Lambda})$, the meson-nucleon interaction $(K \bar{N} \rightarrow \pi \bar{\Lambda})$, and the meson-meson interaction $(M M \rightarrow \Lambda \bar{\Lambda}$ and $K M \rightarrow \bar{\Lambda} N)$, where $M$ denotes either a pion or a rho meson. The first process is important during the initial nonequilibrium stage of heavy ion collisions. Its contribution is similar to that from the $p-p$ collision and will not lead to the enhancement of antilambda production. The second process is unimportant as the number of $\bar{N}$ created in the collision is small. The last one would dominate after the compression stage when many mesons are produced. Because of the Okubo-Zweig-Iizuka (OZI) rule 


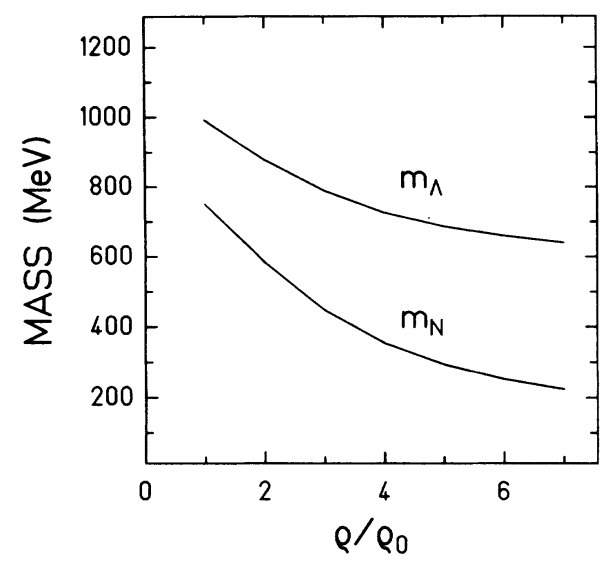

FIG. 1. The density dependence of nucleon and lambda masses from the Walecka model.

[16], the reaction $M M \rightarrow \Lambda \bar{\Lambda}$ is expected to be suppressed and will not be considered. Since there is an appreciable number of kaons produced in the collision, the reaction $K M \rightarrow \bar{\Lambda} N$ is more favorable as it is not suppressed by the OZI rule.

The Feynman diagram for $K M \rightarrow \bar{\Lambda} N$ reaction is shown in Fig. 2. It can be easily evaluated, and the cross section is

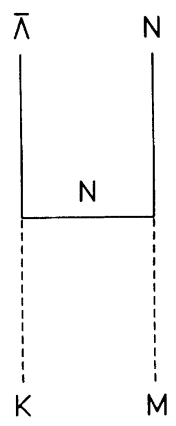

FIG. 2. The Feynman diagram for $K M \rightarrow \bar{\Lambda} N$, where $M$ denotes either a pion or a rho meson.

$$
\sigma_{K M \rightarrow \bar{\Lambda} N}=\frac{p_{f}}{8 \pi s p_{i}} \int_{-1}^{+1} d \cos \theta M(\cos \theta),
$$

where $s$ is the square of the center-of-mass energy. The initial and final three momenta of the particles are denoted by $p_{i}$ and $p_{f}$, respectively, while the angle between them is given by $\theta$.

Using the pseudoscalar coupling for the $\Lambda N K$ and $N N \pi$ vertices, the squared invariant amplitude $M_{\pi}(\cos \theta)$ for the reaction $K \pi \rightarrow \bar{\Lambda} N$ can be expressed as

$$
\begin{aligned}
M_{\pi}(\cos \theta)= & \left(\frac{g_{\Lambda N K} g_{N N \pi}}{q^{2}-m_{N}^{2}}\right)^{2}\left(\frac{\Lambda_{\Lambda N K}^{2}-m_{N}^{2}}{\Lambda_{\Lambda N K}^{2}-q^{2}}\right)^{2}\left(\frac{\Lambda_{N N \pi}^{2}-m_{N}^{2}}{\Lambda_{N N \pi}^{2}-q^{2}}\right)^{2} \\
& \times\left[2 p_{\bar{\Lambda}} \cdot q p_{N} \cdot q-p_{\bar{\Lambda}} \cdot p_{N} q^{2}-m_{N}^{2}\left(2 p_{\bar{\Lambda}} \cdot q-p_{\bar{\Lambda}} \cdot p_{N}\right)+m_{N} m_{\Lambda}\left(2 p_{N} \cdot q-q^{2}\right)-m_{N}{ }^{3} m_{\Lambda}\right]
\end{aligned}
$$

For the $N N \rho$ vertex, we include both the vector and the tensor couplings. The squared invariant amplitude $M_{\rho}(\cos \theta)$ for the reaction $K \rho \rightarrow \bar{\Lambda} N$ is then

$$
\begin{aligned}
& M_{\rho}(\cos \theta)= \frac{1}{3}\left(\frac{g_{\Lambda N K} g_{N N \rho}}{q^{2}-m_{N}^{2}}\right)^{2}\left(\frac{\Lambda_{\Lambda N K}^{2}-m_{N}^{2}}{\Lambda_{\Lambda N K}^{2}-q^{2}}\right)^{2}\left(\frac{\Lambda_{N N \rho}^{2}-m_{N}^{2}}{\Lambda_{N N \rho}^{2}-q^{2}}\right)^{2} \\
& \times\left\{( 1 + \frac { f _ { N N \rho } } { g _ { N N \rho } } ) ^ { 2 } \left[8 m_{N}^{2}\left(p_{\bar{\Lambda}} \cdot q+m_{N} m_{\Lambda}\right)-4 p_{N} \cdot p_{\rho}\left(p_{\bar{\Lambda}} \cdot q+3 m_{N} m_{\Lambda}\right)\right.\right. \\
&\left.-m_{\rho}{ }^{2}\left(p_{\bar{\Lambda}} \cdot p_{N}-3 m_{N} m_{\Lambda}\right)+\frac{4}{m_{\rho}^{2}}\left(p_{\bar{\Lambda}} \cdot p_{N}+m_{N} m_{\Lambda}\right)\left(p_{N} \cdot p_{\rho}\right)^{2}\right]\left.\quad+\frac{4}{m_{\rho}^{2}}\left(p_{N} \cdot p_{\rho}\right)^{2}\left(m_{\Lambda} p_{N} \cdot p_{\rho}+m_{N} p_{\bar{\Lambda}} \cdot p_{\rho}\right)\right\} \\
&-2\left(1+\frac{f_{N N \rho}}{g_{N N \rho}}\right)\left(\frac{f_{N N \rho}}{2 m_{N} g_{N N \rho}}\right)\left\{2 p _ { N } \cdot p _ { \rho } \left[m_{\Lambda}\left(m_{\rho}{ }^{2}-2 m_{N}{ }^{2}-3 p_{N} \cdot p_{\rho}\right)\right.\right. \\
&-\left(\frac{f_{N N \rho}}{2 m_{N} g_{N N \rho}}\right)^{2}\left[\left(\left(q+p_{N}\right)^{2}-\frac{\left(m_{N}{ }^{2}-q^{2}\right)^{2}}{m_{\rho}^{2}}\right)\right. \\
&\left.\left.\quad \times\left[2 p_{\bar{\Lambda}} \cdot q p_{N} \cdot q-p_{\bar{\Lambda}} \cdot p_{N} q^{2}+m_{N}^{2}\left(p_{\bar{\Lambda}} \cdot p_{N}-2 p_{\bar{\Lambda}} \cdot q\right)+m_{N} m_{\Lambda}\left(2 p_{N} \cdot q-q^{2}\right)\right]\right]\right\} .
\end{aligned}
$$

In Eqs. (3) and (4), $p_{\bar{\Lambda}}, p_{N}$, and $p_{\rho}$ are, respectively, the four momenta of the antilambda, the nucleon and the rho meson. The four momentum transfer is given by $q=p_{K}-p_{\bar{\Lambda}}$.
The pseudoscalar coupling constants $g_{\Lambda N K}$ and $g_{N N \pi}$, the vector and tensor coupling constants $g_{N N \rho}$ and $f_{N N \rho}$, and the cutoff parameters $\Lambda$ in the form factor at the vertices are taken from Holzenkamp, Holinde, and Speth 

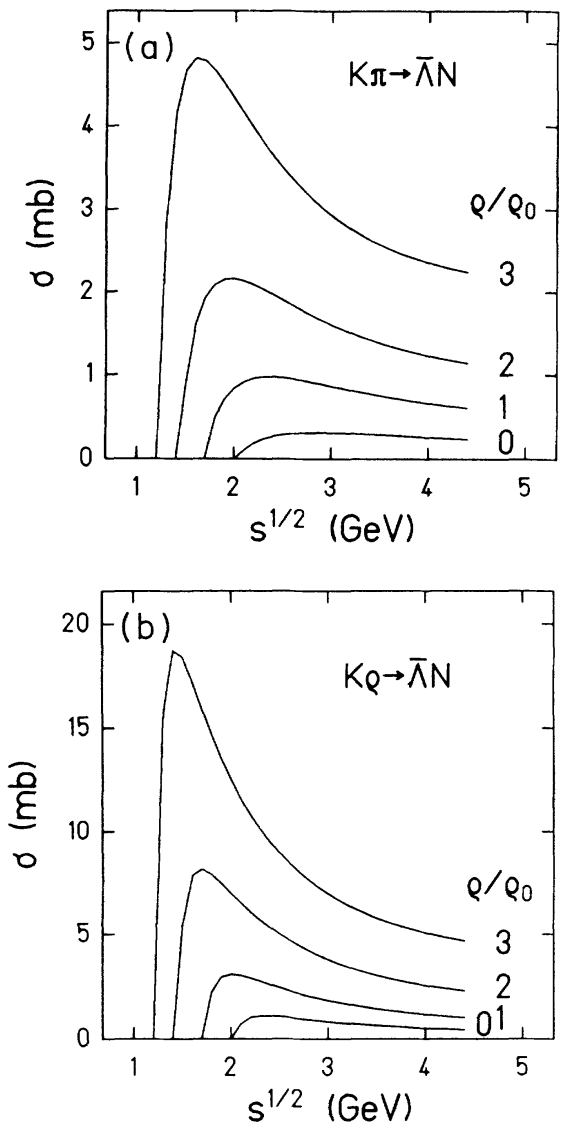

FIG. 3. The energy dependence of (a) $\sigma_{K \pi \rightarrow \bar{\Lambda} N}$ and (b) $\sigma_{K \rho \rightarrow \bar{\Lambda} N}$ at different densities.

[17]. They are $g_{\Lambda N K}^{2} / 4 \pi \approx 15.6, g_{N \pi \pi}^{2} / 4 \pi \approx 14.4$, $g_{N N \rho}^{2} / 4 \pi \approx 0.85, f_{N N \rho}^{2} / 4 \pi \approx 21.3$, and $\Lambda_{\Lambda N K} \sim$ $\Lambda_{N N \pi} \sim \Lambda_{N N \rho} \approx 1.2 \mathrm{GeV}$. The density dependence of the coupling constants and the cutoff parameters cannot be determined in the Walecka model. In the present study, we take the coupling constants to be independent of the density but allow the cutoff parameters to scale with the in-medium masses to describe the increase of hadron sizes as their masses decrease in dense matter [18].

In Fig. 3, the energy dependence of the cross sections $\sigma_{K \pi \rightarrow \bar{\Lambda} N}$ and $\sigma_{K \rho \rightarrow \bar{\Lambda} N}$ are shown for different densities. It is seen that in dense matter not only the threshold of the reaction is reduced but also the magnitude of the cross sections is increased. We note that $\sigma_{K \rho \rightarrow \bar{\Lambda} N}$ is larger than $\sigma_{K \pi \rightarrow \bar{\Lambda} N}$.

For determining the antilambda production rate in hot dense matter, it is of interest to evaluate the thermal average of the product of the antilambda production cross section and the kaon-meson relative velocity $\langle\sigma v\rangle_{K M \rightarrow \bar{\Lambda} N}$. In Fig. 4, this quantity is shown as a function of the temperature for different densities and is seen to increase significantly with the density. Again, we have $\langle\sigma v\rangle_{K \rho \rightarrow \bar{\Lambda} N}$ much greater than $\langle\sigma v\rangle_{K \pi \rightarrow \bar{\Lambda} N}$.

The antilambda yield in nuclear matter can then be calculated from the kinetic equation
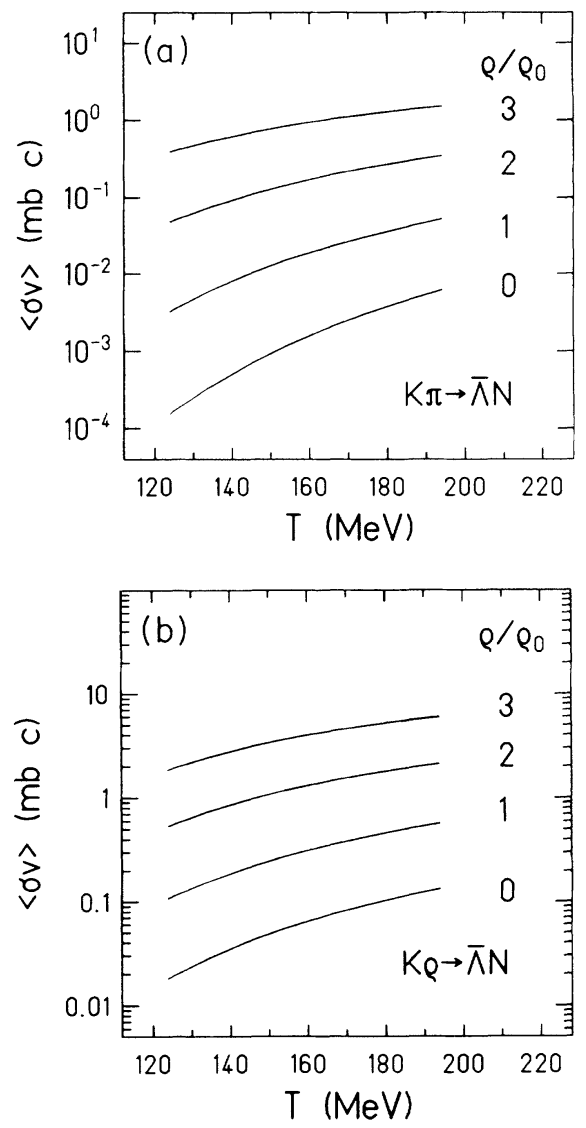

FIG. 4. The temperature dependence of (a) $\langle\sigma v\rangle_{K \pi \rightarrow \bar{\Lambda} N}$ and (b) $\langle\sigma v\rangle_{K \rho \rightarrow \Lambda N}$ at different densities.

$$
\begin{aligned}
\frac{1}{V} \frac{d\left(V \rho_{\bar{\Lambda}}\right)}{d t}= & \langle\sigma v\rangle_{K \pi \rightarrow \bar{\Lambda} N} \rho_{K} \rho_{\pi}\left[1-\frac{\rho_{\bar{\Lambda}} \rho_{N} \rho_{K}^{(0)} \rho_{\pi}^{(0)}}{\rho_{\bar{\Lambda}}^{(0)} \rho_{N}^{(0)} \rho_{K} \rho_{\pi}}\right] \\
& +\langle\sigma v\rangle_{K \rho \rightarrow \bar{\Lambda} N} \rho_{K} \rho_{\rho}\left[1-\frac{\rho_{\bar{\Lambda}} \rho_{N} \rho_{K}^{(0)} \rho_{\rho}^{(0)}}{\rho_{\bar{\Lambda}}^{(0)} \rho_{N}^{(0)} \rho_{K} \rho_{\rho}}\right]
\end{aligned}
$$

where $\rho$ 's and $V$ are, respectively, the time-dependent particle densities and volume of the nuclear matter. The equilibrium densities are denoted by $\rho^{(0)}$ 's. As in Refs. [5, $8]$, the expansion of the hadronic matter is described by a simplified hydrodynamical equation with linear scaling ansatz for the velocity profile. The hadronic matter is assumed to be in thermal equilibrium and the thermal energy is converted to flow energy as the system expands.

For the collision between two sulfur nuclei at 200 $\mathrm{GeV} /$ nucleon, the Lorentz factor in the center of mass is about 10 and it is thus expected that a fire-cylinder is formed in the collision [8]. Its cross section is given approximately by $\pi R^{2} \approx 40 \mathrm{fm}^{2}$, where $R \approx 3.5 \mathrm{fm}$ is the radius of the sulfur nucleus. The number of baryons in the fire-cylinder can be inferred from the measured nucleon distribution in the central rapidity [11], $d N / d y \approx 6$, to be about 36 . The initial density is expected to be high [19] and is taken to be $2 \rho_{0}$. Then the initial longitudinal 


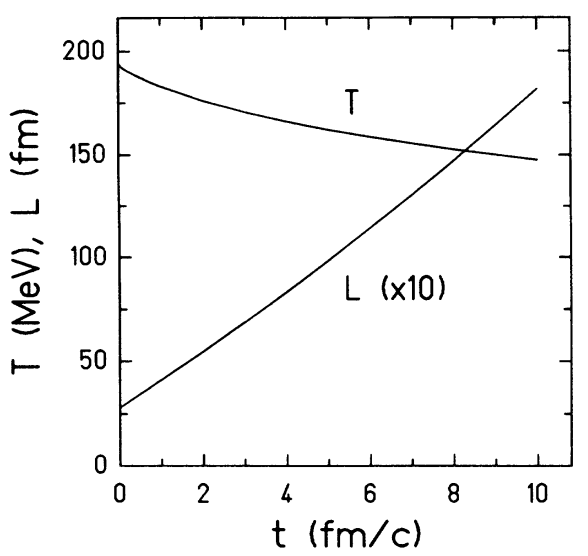

FIG. 5. The time dependence of the temperature and length of the fire-cylinder.

length of the fire-cylinder is about $3 \mathrm{fm}$. The initial temperature of the system should also be quite high and we take it to be $195 \mathrm{MeV}$, slightly higher than that of Ref. [8] in which the sulfur nucleus collides with a uranium target.

The masses of nonstrange hadrons are assumed to decrease in hot dense matter as the nucleon [20]. Their interactions are expected to be enhanced and so they are assumed to be in chemical equilibrium. But the strange hadrons, including both kaons and lambdas as well as their antiparticles, are not in chemical equilibrium and their abundance is determined by rate equations. Kaons and antikaons are mainly produced in the hadronic matter from the meson-meson interaction $M M \rightarrow K \bar{K}$. The lambda particle is produced from the absorption of an antikaon by a nucleon via the reaction $\bar{K} N \rightarrow \Lambda \pi$. The rate equations for these particles and the corresponding $\langle\sigma v\rangle$ can be found in Ref. [5].

The results of the hydrochemical calculations are shown in Figs. 5 and 6 . In Fig. 5, the time dependence of the temperature of the fire cylinder and its length is shown. The density of the system is reduced to one third of the normal nuclear matter density after about $10 \mathrm{fm} / c$ when the temperature is about $150 \mathrm{MeV}$. We take this as the freeze out conditions. The time evolution of the abundance of nonstrange hadrons is shown in Fig. 6(a). We see that at freeze out the number of thermal pions is about 85 while the number of pions from the decays of both rho mesons and baryon resonances is about 165 . Altogether we have about 250 pions, and this number is slightly smaller than the $\sim 300$ pions measured in the CERN experiment. The time dependence of the abundance of strange hadrons is shown in Fig. 6(b). The final kaon number is about 14 and again is smaller than the measured number of $\sim 20$ kaons. The lambda number is about 4 and is less than the measured number of $\sim 8$. The smaller numbers of pions, kaons, and lambdas from our model, which are fewer than the measured ones, may be attributed to particle production from the
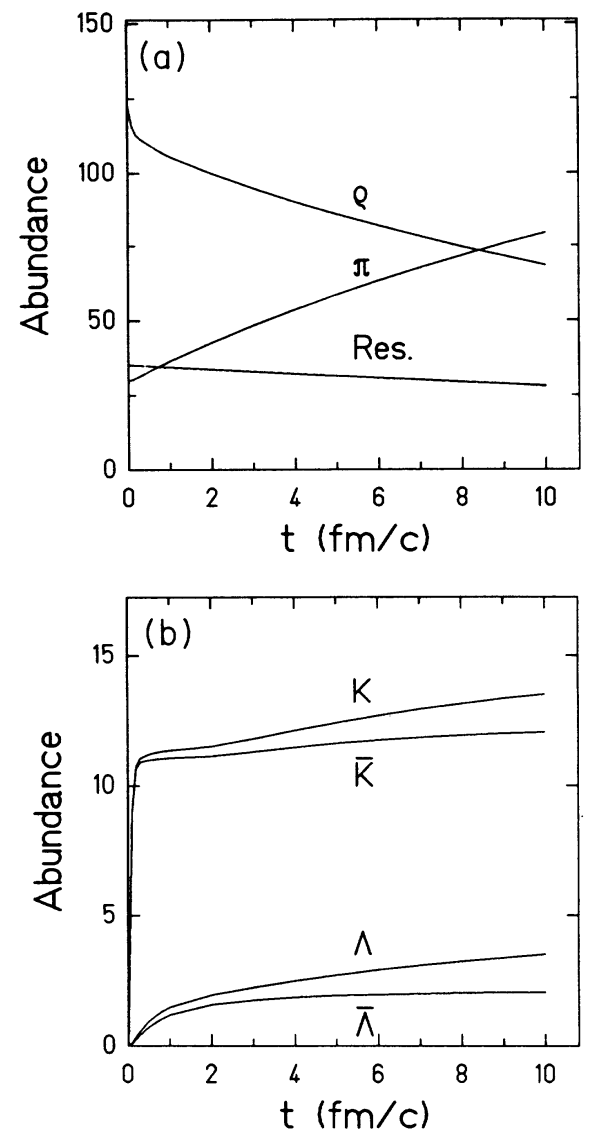

FIG. 6. The time dependence of the abundance of (a) nonstrange and (b) strange hadrons.

initial nonequilibrium stage and from the spectator matter which has been ignored in our study. The antilambda number is about two and is comparable to the measured value of $1.5 \pm 0.4$.

In conclusion, we have obtained enhanced production of antilambdas in hot and dense matter if the decreasing in-medium hadronic masses are included. The enhanced antilambda production measured in ultrarelativistic heavy ion collisions at the CERN energy thus offers the possibility to study the property of hadrons in dense matter formed in the compression stage of the collision. This study is also important for future heavy ion experiments at higher energies where the quark-gluon plasma is expected to be formed in the collisions. To find the signatures for the formation of the quark-gluon plasma, it is essential to have a good understanding of the hadronic physics which exists both in the initial and later stages of heavy ion collisions. Further work is therefore needed to understand the relative importance of the different mechanisms for antilambda enhancement.

This work was supported in part by the National Science Foundation under Grant No. PHY-8907986 and the Welch Foundation under Grant No. A-1110. 
[1] Quark Matter '90, Nucl. Phys. A525, 1c (1991), and references therein.

[2] J. Rafelski and B. Müller, Phys. Rev. Lett. 48, 1066 (1982).

[3] R. Mattiello, H. Sorge, H. Stöcker, and W. Greiner, Phys. Rev. Lett. 63, 1549 (1989).

[4] P. Koch, U. Heinz, and J. Pisút, Phys. Lett. B 243, 149 (1990).

[5] C. M. Ko, Z. G. Wu, L. H. Xia, and G. E. Brown, Phys. Rev. Lett. 66, 2577 (1991); Phys. Rev. C 43, 1881 (1991).

[6] G. E. Brown, C. M. Ko, and K. Kubodera, Z. Phys. A 341, 301 (1992)

[7] T. Abbott et al., Phys. Rev. Lett. 64, 847 (1991).

[8] C. M. Ko and B. H. Sa, Phys. Lett. B 258, 6 (1991).

[9] C. M. Ko, P. Lévai, X. J. Qiu, and C. T. Li, Phys. Rev. C 45, 1400 (1992).

[10] J. P. Guilland et al., Nucl. Phys. A525, 499c (1991).

[11] J. Bartke et al. (NA35 Collaboration), Z. Phys. C 48, 191 (1990); R. Stock et al. (NA35 Collaboration), Nucl.
Phys. A525, 221c (1990).

[12] J. Aichelin and K. Werner, University of Heidelberg Report HD-TVP-91-18.

[13] H. Sorge, M. Berenguer, H. Stöcker, and W. Greiner, Los Alamos Report LA-UR-92-1078.

[14] N. K. Glendenning and S. A. Moszkowski, Phys. Rev. Lett. 67, 2414 (1991).

[15] C. H. Johnson, D. J. Horen, and C. Mahaux, Phys. Rev. C 36, 2252 (1987).

[16] For a review, see S. Okubo, Prog. Theor. Phys. Suppl. 63, 1 (1978).

[17] B. Holzenkamp, K. Holinde, and J. Speth, Nucl. Phys. A500, 485 (1989).

[18] R. Pisarski, Phys. Lett. 110B, 155 (1982).

[19] H. Sorge, A. von Keitz, R. Mattiello, H. Stöcker, and W. Greiner, Nucl. Phys. A525, 95c (1991).

[20] G. E. Brown and M. Rho, Phys. Rev. Lett. 66, 2720 (1991). 